\title{
Gestión pública y ciudadanía política, una aproximación teórica
}

Francisco Sorto Rivas*

Facultad de Maestrías y Estudios de Postgrado, UTEC

\section{Resumen}

Este artículo es la reseña de una investigación que será publicada por la Universidad Tcenológica de El Salvador, UTEC, este año. En él se sistematiza el desarrollo teórico de las últimas décadas en materia de gestión pública, cuyo diseño y ejecución responde a las necesidades prioritarias del colectivo imaginario desde el ámbito de actuación de las instituciones públicas, de tal manera que su intervención en la organización social para zanjar carencias colectivas sea apreciada por la ciudadanía en general y por los usuarios de estas en particular.

El diseño de los planes, programas, proyectos y productos externos estarán mejor alineados a las necesidades colectivas en la medida que los ciudadanos y usuarios dispongan de los espacios públicos que les permitan expresar cuáles son las necesidades colectivas urgentes que requieren de soluciones de política.

\section{Palabras claves:}

Gestión pública, gobierno corporativo, planeamiento estratégico, rendición de cuentas, ciudadanía política.

\section{Metodología}

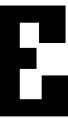

sta investigación se realizó mediante la revisión de bibliografía reciente acerca de gestión pública, a lo largo de los últimos tres años en que el autor ha sido docente asesor del programa de Postgrado: “Planificación y Rendición de Cuentas por Resultados y Tablero de Control", impartido desde la modalidad de educación a distancia por la Universidad Nacional del Litoral de Argentina.
Esta investigación fue iniciada a partir de las contribuciones de los profesores Óscar Oszlak y Jorge Hintze, en el seno del CLAD, y por el interés profesional del autor de abordar, de manera ordenada, las experiencias de más de 20 años en la administración pública, en distintas dependencias del Estado, donde ha podido comprobar la validez de los aportes presentados en este documento $y$, además, influenciada por su labor como investigador y consultor de varios organismos internacionales como el BID, el Banco Mundial, Cepal, Unctad, PNUD, Prodere y US-AID.

Es una investigación descriptiva y prospectiva, a la vez. Se desarrolló a lo largo de dos años y es eminentemente bibliográfica y reflexiva. Un estudio teórico que Montero y León (2007) lo clasifica como metaanálisis.
Economista, Master en Economía Empresarial de INCAE, Costa Rica y especialista en Gestión de Políticas Públicas de la Universidad Nacional del Litoral, Argentina. Docente, Facultad de Maestrías y Postgrados.

El autor agradece los comentarios del doctor Jorge Hintze sobre algunos apartados del presente trabajo, así como la colaboración de los licenciados Silvia América González y José Rodolfo Castillo, que revisaron todo el documento; gracias a sus apreciados comentarios este documento fue enriquecido significativamente. Igual agradecimiento merece la labor de la señora Zuleima Mendoza, que se encargó de integrar buena parte de los apuntes originales de este documento.

Se agradece además a la Universidad Tecnológica de El Salvador, y en especial a la licenciada Blanca Ruth Orantes, por su interés en apoyar al cuerpo docente de esta universidad en el campo de la investigación científica. 


\section{Introducción}

Para comenzar este ejercicio cabe destacar que, al hacer referencia a la gestión pública en muchos países de América Latina, el lector podria tender a predisponerse automáticamente y pensar que tendrá que revisar conceptos convencionales de administración pública, donde prima la descripcióndeprocedimientosrígidos (burocráticos), inefectivos para lograr las metas operativas trazadas por la dirección e ineficientes en la gestión de los recursos utilizados para su funcionamiento.

Se tiende a creer también que, al hablar de este tipo de temas, se van a analizar, irremediablemente, estructuras jerarquizadas al extremo, propias de organizaciones piramidales, donde la toma de decisiones responde a una cadena de mando formal e inflexible y donde el personal está más enfocado hacia el proceso descrito en un manual que en las necesidades que tienen los usuarios de las políticas públicas, que se deben gestionar a través de este tipo de organizaciones.

Por otro lado, cabe destacar que aún existen casos en que los encargados de la gestión de algunas instituciones públicas son incapaces de identificar quiénes son sus usuarios naturales, qué productos

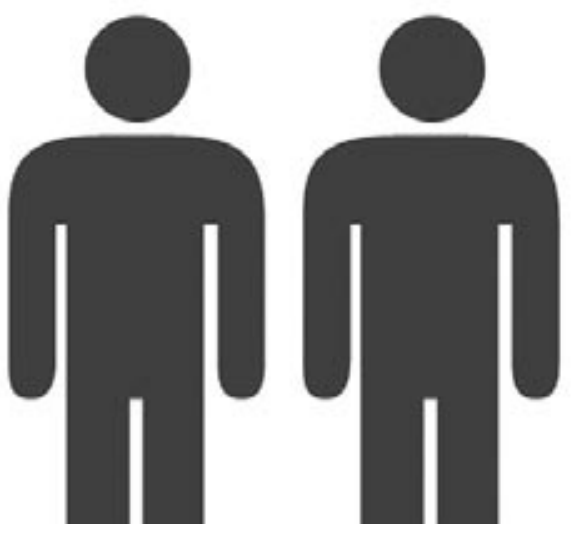

\section{La efectividad en}

la gestión de fondos

por parte de las insti-

tuciones públicas

se convierte, entonces,

en un imperativo para

un buen gobierno

corporativo, ya que el

ciudadano se convierte

en parte de

la contraloría social

del sistema concebido

para mantener

la cohesión social y

el sentido de pertenencia

de los ciudadanos al

Estado.

externos deben diseñar para ellos, siguiendo ciertos principios de eficiencia y economía de recursos, permaneciendo siempre alineados con la misión y visión institucional. Todo esto a pesar del desarrollo teórico registrado sobre la materia en las últimas tres o cuatro décadas.

Normalmente se asume que este tipo de literatura está centrada en

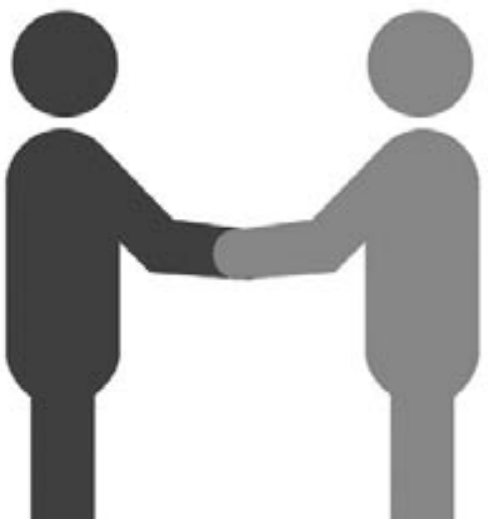

la descripción de la organización del Estado, la división de poderes, la constitución de instituciones públicas, sus competencias y mecanismos de control de la gestión financiera; sin embargo, el enfoque epistemológico de esta investigación gira en torno a la ciudadanía política y la contribución de una gestión moderna al perfeccionamiento de este concepto.

Es más, muchas personas -incluyendo empleados y funcionarios públicos - parecen ignorar que dichas instituciones responden a un propósito particular y que, al igual que toda organización social, están sujetas a cambios, reingeniería, redefinición de funciones e, inclusive, hasta a la desaparición.

Tampoco se discute mucho sobre el valor público que estas deben crear y a partir del cual se deberían evaluar, a lo largo de un ejercicio fiscal, programa o proyecto. De hecho, es rara la ocasión cuando lo hacen de forma independiente. De ahí la importancia de la rendición de cuentas, pero no la convencional centrada únicamente en la revisión de la ejecución presupuestaria, sino en el análisis de la estructura de gastos y cómo esta se traduce en beneficios para el imaginario social relevante para la organización, según las políticas que le toque administrar.

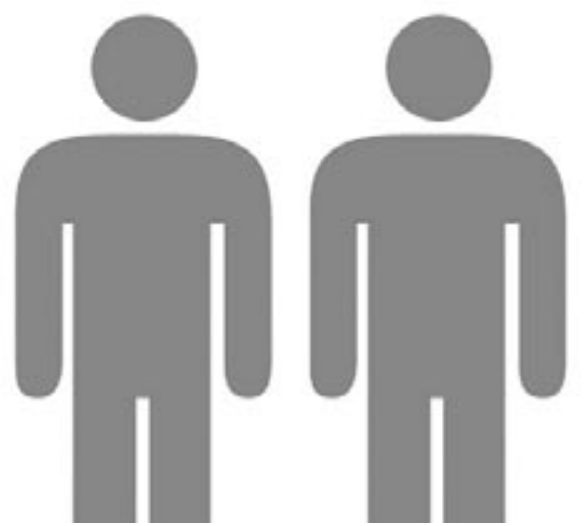


Se tiende a

creer también que,

al hablar de este tipo

de temas, se van a

analizar, irremediable-

mente, estructuras

jerarquizadas al

extremo, propias

de organizaciones

piramidales, donde

la toma de decisiones

responde a una cadena

de mando formal e

inflexible.
En el marco de la nueva gestión pública (NGP), resulta inconcebible pensar en las instituciones públicas como fines en sí mismas; su existencia obedece a una necesidad colectiva que requirió la participación del Estado para resolverla, y cuyo diseño quedó recogido en una ley, siendo su expresión material una institución gubernamental encargada de administrar ciertas políticas resultantes del compromiso asumido con actores representativos de los afectados por cierta problemática común. En el documento se destaca la importancia que tiene, para la gobernabilidad y legitimidad de las políticas, el compromiso de las instituciones del Estado hacia la sociedad en general y hacia sus usuarios en particular, lo cual se aprecia a través de la eficiencia, eficacia y economía de los recursos asignados para su funcionamiento

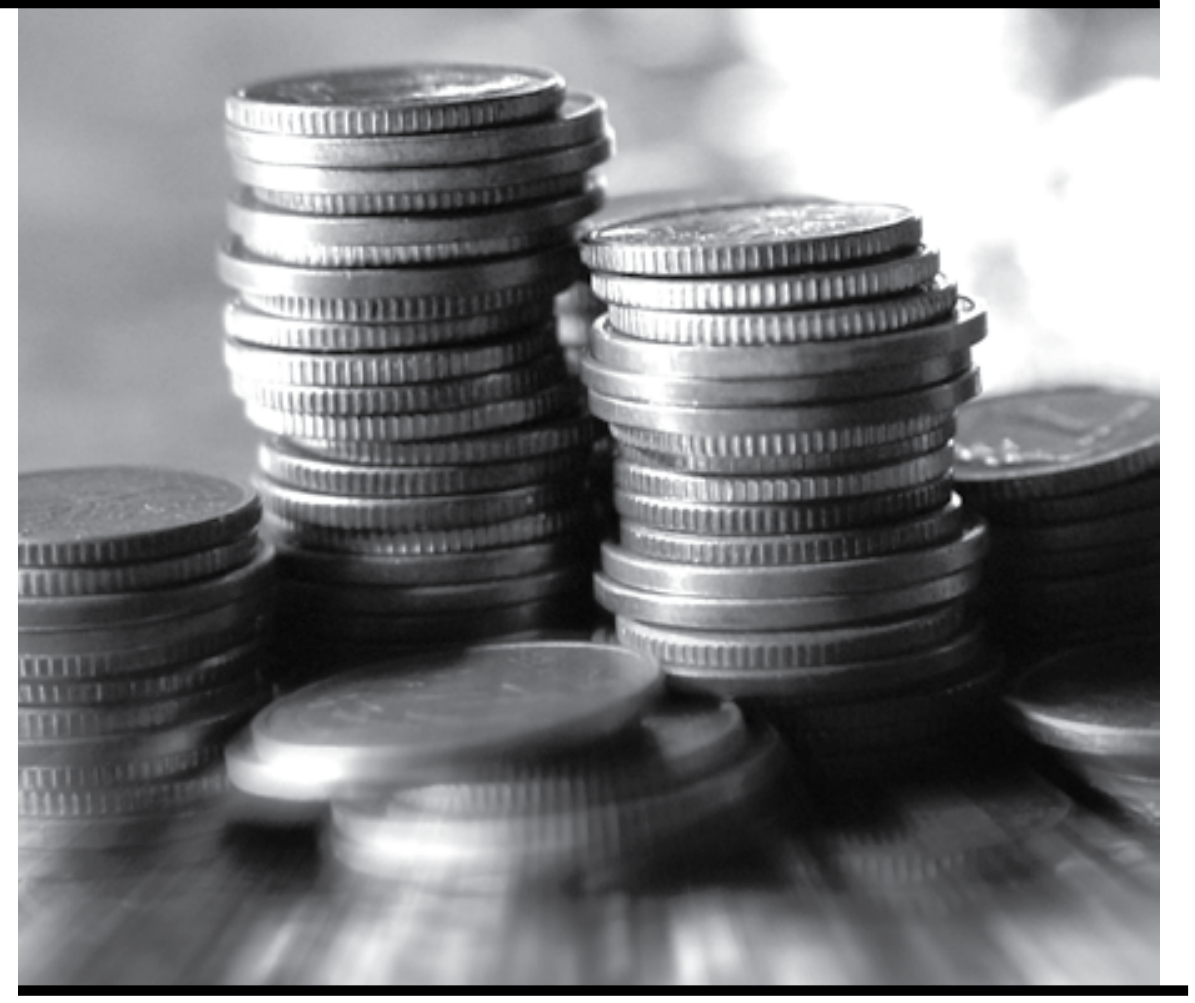

y cómo estos se traducen en bienes, servicios o símbolos, a manera de contrapartida por los impuestos, tasas o contribuciones recolectadas.

En el documento se demuestra que la rendición de cuentas es un atributo indispensable para la legitimidad del gasto, debiendo responder, por supuesto, a un plan estratégico donde se alineen la misión, visión, objetivos estratégicos institucionales y los productos externos creadores de valor público para la sociedad. La efectividad en la gestión de fondos por parte de las instituciones públicas se convierte, entonces, en un imperativo para un buen gobierno corporativo, ya que el ciudadano se convierte en parte de la contraloría social del sistema concebido para mantener la cohesión social y el sentido de pertenencia de los ciudadanos al Estado. La provisión de bienes y prestación servicios meritorios que son su responsabilidad puede hacerse directamente a través de instituciones públicas o mediante algún nivel de descentralización e, inclusive, por medio de la concesión total o parcial a terceros.

Cuando se delega la prestación de servicios públicos a entidades privadas, las funciones públicas deben reorganizarse para readecuar el uso de los recursos destinados originalmente a la provisión pública de estos; es así que deberán orientarse a labores de regulación, fiscalización y control de los operadores privados, la divulgación de los derechos de los usuarios, la sanción por algún tipo de deterioro en la calidad de los servicios y, eventualmente, la remoción de las licencias de operación que fueron otorgadas inicialmente a las firmas, 
cuando estas no corrijan los defectos señalados.

Dicha actuación está

orientada a preservar el control de los resultados esperados del diseño e implantación de una política pública que, teniendo como parte del diseño la provisión de servicios, el Estado hubiera decidido concesionarla por razones de eficiencia y economía de recursos. Es por ello que los recursos públicos deberán organizarse en consecuencia, para cumplir eficazmente con los objetivos estratégicos trazados en el ámbito de Gobierno, en beneficio de sus ciudadanos y residentes bajo su jurisdicción, indistintamente de la figura utilizada para la entrega de los servicios. Por otro lado, es necesario señalar aquí que la NGP no es una escuela de pensamiento administrativo claramente definido, ni está perfectamente estructurada, aunque exista abundante literatura sobre planeación estratégica para instituciones públicas y para el alineamiento de esta con sus misiones y visiones, así como con los productos externos por entregarse a los usuarios. Adicionalmente, este ejercicio de gestión moderna de la burocracia supone una mejora sustancial en la asignación responsable de los recursos públicos, ya que con esta herramienta se definen programas y proyectos en beneficio de los usuarios, mediante la priorización de acciones y necesidades y, a su vez, se convierten en parámetros para la evaluación del desempeño de las organi-zaciones y sus directorios.
En la medida que se dispone de espacios públicos adecuados, los ciudadanos pueden manifestar eficientemente qué opinan sobre la gestión de las instituciones $y$, con ello, incidir positivamente sobre la calidad de los servicios prestados, avanzando de paso hacia una mayor legitimidad del trabajo realizado y a la percepción de que el Gobierno se preocupa por el bien común. De igual manera hay políticas de comunicación hacia el exterior que perjudican la efectividad del trabajo institucional, ya que las personas que están fuera de la organización no saben, posiblemente, cuáles son las competencias que le han delegado, por lo que los usuarios puedan formarse expectativas inapropiadas sobre cuál es la razón que recordar que, a diferencia de intercambio convencional de bienes y servicios privados, donde el cliente puede incidir instantáneamente sobre la gestión del proveedor al decirle, mediante sus decisiones de compra, si concuerda con sus expectativas o no. Cuando se trata de bienes y servicios públicos, la relación de intercambio puede prescindir del pago como contraprestación entre consumidores y proveedores, de tal manera que no se dispone de mecanismos de retroalimentación instantánea. Esto obliga a las instituciones públicas a recurrir a encuestas sobre el grado de satisfacción de sus usuarios, como alternativa ante la ausencia de retroalimentación mediante el intercambio con los usuarios, o a establecer algún otro mecanismo que le permita a estos expresar su conformidad o no con el trabajo realizado por las instituciones estatales.

\section{En el marco}

\section{de la nueva gestión}

pública (NGP), resulta

inconcebible pensar en

las instituciones públicas

como fines en sí mismas;

su existencia obedece

a una necesidad colec-

tiva que requirió la

participación del Estado

para resolverla, y cuyo

diseño quedó recogido

en una ley. $y$ 
de su existencia. Se puede decir, además, que la profesionalización del recurso humano también es objeto de estudio en el marco de la NGP, dado que es poco lo que se puede lograr en materia de mejoras cuando se dispone de poco capital humano. De hecho, al hablar de la reforma administrativa del Estado, siempre se enfatiza en la necesidad de implantar planes de carrera que les dé estabilidad a los funcionamientos en el aparato público, aunque se experimente cambios de Gobierno; esta es, entonces, una condición sine qua non para elevar la gestión de la cosa pública: contar con profesionales competentes, motivados y con conocimientos suficientes para administrar eficientemente los recursos colectivos.

De igual manera, contar con recurso calificado pero con una dirección deficiente puede traducirse en un pobre desempeño; así que el tema del liderazgo también constituye un tema de estudio para la NGP. El estilo de liderazgo es vital para la instauración de un entorno de trabajo disciplinado

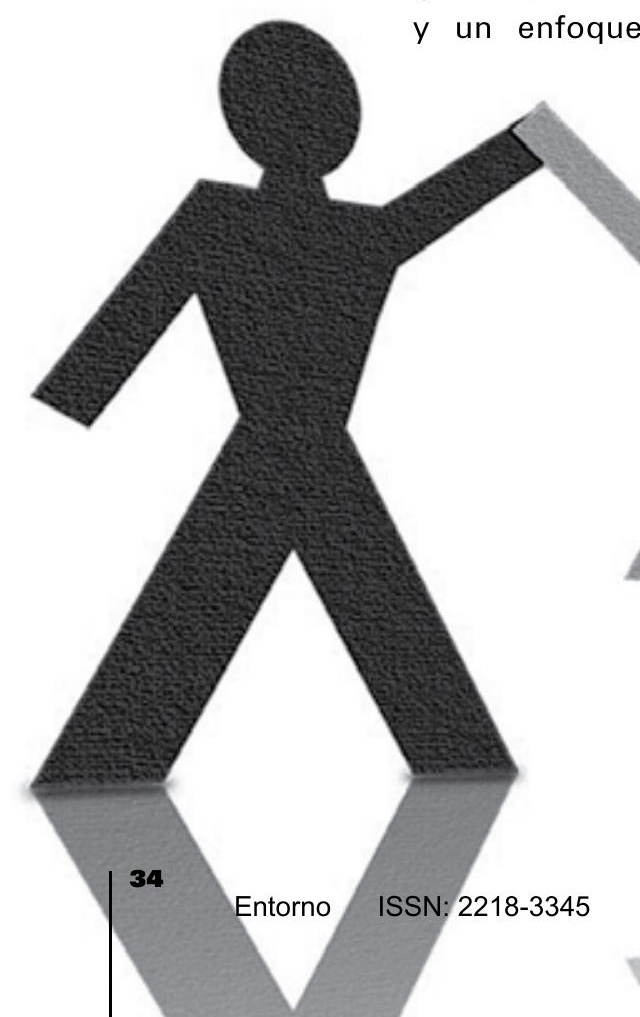

responsable hacia el cumplimiento de metas, los procesos y los clientes; la confianza que pueda construirse para delegar tareas rutinarias le puede permitir a las autoridades de una institución destinar su tiempo a fijar el rumbo de la organización; caso contrario, se verá inundado por todo tipo de decisiones que le absorberán su tiempo, sin permitirle desarrollar todo su pensamiento estratégico en la institución. Cuando esto ocurre, al no disponer de tiempo para analizar sopesadamente los asuntos sobre los cuales tiene que tomar decisiones, termina entregándole la agenda estratégica a los subalternos, quienes acaban usurpando, de alguna manera, consciente $o$ inconscientemente, sus facultades para dirigir la institución, dentro de las cuales se encuentra la autoridad para establecer prioridades y la orden del día.

Introduzcamos ahora una definición propuesta por Blanca Olías de Lima, sobre el tema que nos ocupa -NGP-: "la nueva gestión pública es el intento de dar cuenta, reflexionar y discutir sobre cómo alcanzar fines públicos con la mayor eficiencia, eficacia y satisfacción del ciudadano". De esta propuesta epistemológica se pueden extraer algunas ideas básicas sobre el tema: 1) que mediante esta concepción se enfatiza la búsqueda de fines públicos; 2) que al hacerlo, la gestión debería estar mediatizada por la eficacia y la eficiencia; y 3) que la gestión debe orientarse a la satisfacción de necesidades ciudadanas.

En esta definición, el uso del término ciudadano no es antojadizo, sino que se utiliza para referirse a la persona como ente político, consciente de sus derechos y responsabilidades, con una actitud crítica hacia los problemas colectivos y una disposición clara a participar en su debate y discutir posibles soluciones. De ahí se desprende el grado de gobernabilidad detentado en un momento determinado por las autoridades estatales.

A partir de esta realidad, parece lógico afirmar que la gestión de una política pública no necesariamente debe hacerse mediante una institución pública, aunque es imprescindible que exista una que se encargue de velar por el cumplimiento de los objetivos que motivaron la promulgación de la ley. 


\section{Conclusiones}

La gestión pública en El Salvador está transitando a diferentes velocidades, y bajo diversos enfoques, hacia una gestión orientada a resultados, abandonando la gestión convencional de gestión financiera y de cumplimiento. Aunque falta mucho camino por recorrer. El planeamiento estratégico es poco utilizado aún por la mayoría de instituciones públicas en El Salvador, especialmente en las carteras de Estado y gobiernos locales. La rendición de cuentas es una tarea más formal que real en el país, dado que no está integrada aún a la evaluación del desempeño de las instituciones y sus directorios, ni sirve de información relevante para la toma de decisiones acerca de la asignación de recursos, responsables y tareas dentro de la administración pública.

La labor de coordinación asociada con el trabajo de control y rendición de cuentas requiere ser reforzado, dado que existe una distribución de competencias entre diversas instituciones, las cuales corresponden a un ámbito organizacional común, pero cuyas acciones individuales no se coordinan adecuadamente para obtener la sinergia necesaria para un uso eficiente de los recursos públicos. Todavía resulta deficiente la alineación entre los productos externos generados por las instituciones públicas y las necesidades y aspiraciones de los usuarios, lo cual abre espacios de mejora importantes para avanzar hacia una mayor legitimidad, gobernabilidad y creación de valor público, en la medida que se vayan resolviendo esas deficiencias $y$ ampliado, paralelamente, los espacios públicos para el debate y la retroalimentación social acerca del desempeño de las organizaciones del Estado.

La ciudadanía política no constituye aún un elemento central para el diagnóstico de las necesidades colectivas, el diseño y la ejecución de medidas de política, así como para la evaluación del desempeño de las instituciones públicas encargadas de su ejecución. De hecho, el tratamiento que se le da a la participación ciudadana dentro de esta lógica es la de simple cliente, lo cual pone en riesgo el respeto de su calidad de derechohabiente dentro de la sociedad. cac Cuando se trata de bienes y servicios públicos, la relación de intercambio puede prescindir del pago como contraprestación entre consumidores y proveedores, de tal manera que no se dispone de mecanismos de retroalimentación instantánea. Esto obliga a las instituciones públicas a recurrir a encuestas sobre el grado de satisfacción de sus usuarios, como alternativa ante la ausencia de retroalimentación mediante el intercambio con los usuarios

\section{BIBLIOGRAFÍA}

- Arellano Gault, David, Gestión estratégica para el sector público, del pensamiento estratégico al cambio organizacional, Fondo de Cultura Económica, México 2004.

- Hintze, Jorge, Evaluación de resultados, efectos e impactos de valor público, biblioteca virtual de Tecnología para la Organización Pública (TOP). http://www.top.org.ar/documentos/HINTZE\%20 Jorge $\% 20-\% 20$ Evaluacion\%20de $\% 20$ resultados $\% 20$ efectos $\% 20$ e\%20impactos\%20de\%20valor\%20publico.pdf

- Montero, Ignacio y Orfelio, G. León (2007), A guide for naming research studies in Psychology. Vo. 7, No. 3. pp.847-862
- Olias De Lima, Blanca, La Nueva Gestión Pública, Prentice Hall; España 2001.

- Porter, Michael, Ventaja Competitiva, CECSA, México 1988.

- Quiroga, Hugo, Villavicencio, Susana y Vermeren, Patrice (compiladores), Filosofías de la Ciudadanía; HomoSapiens ediciones, Argentina 2001.

- Moore, Mark H. Gestión Estratégica y Creación de Valor en el Sector Público, Paidós, España 1998. 